\title{
Unusual sexual behaviour associated with female depression
}

\author{
M Chandradasa, KALA Kuruppuarachchi
}

DOI: http://doi.org/10.4038/sljpsyc.v8i1.8137

\section{Abstract}

Females have a higher prevalence of depression and more atypical depressive symptoms than men. Depressive disorder and antidepressant therapy is characte-ristically associated with sexual dysfunction, but less so in women than men. Reports of heightened sexual desire during depression are uncommonly reported. Here we describe two Sri Lankan females presenting with unusual sexual behaviour associated with depression. First is a middle-aged woman who complained of recent onset voyeuristic behaviour. Second is a young girl, who was brought by her parents after she went repeatedly missing from her home. She had been trying to seek an intimate relationship with a young man. In both patients, the heightened arousal subsided with the remission of depression. Further studies are required to explore this unusual presentation.

\section{Declaration of interest}

The authors declare that there is no conflict of interest.

Key words

Depression, sexual behaviour, female, voyeurism, Sri Lanka

SL J Psychiatry 2017; 8(1): 32-34

\section{Introduction}

Women have a higher risk of developing depressive disorder compared to men and it is debated that this increased prevalence is due to probable environmental and biological factors. For example, women are recognised to be more help seeking regarding their health problems compared to men (1). In addition depressive disorder has been found to be associated with different female reproductive stages and there is evidence that ovarian hormonal levels play a role in depressive disorder among women of postmenopausal age (2).

Presentation of depressive disorder is associated with cultural beliefs of the relevant community. This means depressive symptoms may present in unusual ways, which may be different to the characteristic patterns described in diagnostic criteria and standard text books. Depression presenting with genital symptoms has been reported from Sri Lanka, in which patients have complained of unusual vaginal and urethral sensations (3). In addition depressive disorder may present as culture bound syndromes such as Dhat and it appears that current diagnostic guidelines do not provide appropriate criteria in regard to these unusual cultural presentations (4).

Studies have found that atypical symptoms of depressive disorder such as fatigue and hypersomnia are more prevalent in women compared to men. In contrast reporting of decreased libido by men appears to be higher compared to that of women. When considering gender differences, factors such as the treatment response and adverse side effects of chemotherapeutic agents cannot be ignored (1).

Sexually disinhibited behaviour confined to depressive episodes in women is somewhat uncommonly reported. Here we describe presentations of two females from Sri Lanka with unusual sexual behaviour, whom were diagnosed of depressive disorder subsequently.

\section{Methods}

Two females were assessed via structured clinical interviews as outpatients by a consultant psychiatrist and diagnosed to have a depressive disorder according to the ICD-10 criteria. Informed written consent was obtained from the first patient and the mother of second patient with assent from the girl.

\section{Case 1}

A 45 year old married female reported recent onset voyeuristic behaviour for about two to three months period. She described that she had recurrent urges to observe other adults undress and have sexual interactions. She had acted upon these urges on few occasions. Her heightened sexual behaviour was not limited to this and she demanded her partner to have more frequent sexual intercourse with her. There was no past history of any mood disorder or paraphilic disorder. The onset of this change in behaviour was accompanied by persistent low mood, lethargy and suicidal ideation and depressed mood on mental state examination. She was diagnosed to have a moderate 
depressive episode and commenced on sertraline. The increased need for sexual pleasure disappeared with reduction of depressive symptoms. She continued her medications for a period of nine months after reaching remission and did not show any recurrence of voyeuristic behaviour.

\section{Case 2}

A 16 year old school girl was brought by her parents after she went missing from her home for a period of one day. According to her, she had left home without planning, in distress. She had met a young man ten years older to her while travelling in the bus. They had started chatting and subsequently he had invited her to his residence, to which she complied without any hesitation. According to her they had fondled each other, but denied any penetrative sex. After spending hours in his place, the man had phoned her parents about her whereabouts and they have come to pick her up. There had been no similar or other sexually risky behaviour by her in the past.

The parents were bewildered by this act and decided to take her for specialist mental health opinion. They revealed that before this act, they have observed her to be increasingly irritable towards others. She had less interactions with them compared to before and was seemingly suffering from reduced energy and recurrent physical complaints such as headaches. The girl reported a subjectively higher sexual desire during the past few weeks, which coincided with the onset of depressive symptoms.

On clinical assessment she had features of a moderate depressive episode including low mood, anhedonia, irritability and depressed mood on mental state examination. According to her parents, such sexually active behaviour was uncharacteristic of her. She was commenced on sertraline and the increased sexual desire and related behaviour subsided. This returned on defaulting medication, where she recommenced approaching male strangers for sexual pleasure. The sexually disinhibited behaviour diminished on recommencing the antidepressant.

\section{Discussion}

The case reports describe unusual sexual behaviour associated with depression in two Sri Lankan females. The first is a middle-aged woman presenting with voyeuristic behaviour confined to a depressive episode. The second is a adolescent girl presenting with highly risky sexual behaviour in the context of a depressive disorder. It is possible that sexual pleasure was sought as a form of relief or comfort during this period of distress. The subsiding of sexual desire and behaviour with the improvement of depression was indicative of a potential association.
In relevance to the first patient, we identified one previous case report on voyeuristic disorder presenting with depression to clinical services (5). We could not access any other publication on voyeuristic behaviour confined to depressive episodes. However increased sexual function has been detected and reported in a male patient during a bipolar depressive episode (6). In relevance to the second patient, there is evidence that adolescent females with symptoms of depression are more likely to engage in risky sexual behaviours. This has been shown in the youth risk behaviour surveillance conducted among high school girls in the United States (7).

In contrast to the two females described here, it has been found that women who suffer from depression have a greater sexual dysfunction compared to that of nondepressed females in the population and the degree of dysfunction has a relationship to the severity of mood disorder (8). In a prospective study, higher levels of anhedonia in females were found to be associated with reduced sexual desire on the subsequent day (9).

With regard to treatment, both patients had an increased need for sexual pleasure prior to the commencement of antidepressants. In contrast there are reports of heightened libido in women, which was seen when they were treated with antidepressants such as fluvoxamine (10).

We hope these case reports would assist other clinicians assessing and treating females with depressive disorder in different cultural settings. Further research is required to explore this uncommonly reported association.

\section{Conflict of interest}

None

M Chandradasa, KALA Kuruppuarachchi, Department of Psychiatry, University of Kelaniya, Ragama

Corresponding author: $\mathrm{M}$ Chandradasa

Email: miyuruc@kln.ac.lk

http://orcid.org/0000-0002-1873-8228

\section{References}

1. Kim JH, Cho MJ, Hong JP, Bae JN, Cho SJ, Hahm BJ, et al. Gender differences in depressive symptom profile: results from nationwide general population surveys in Korea. J Korean Med Sci. 2015; 30(11): 1659-66.

2. Harsh V, Meltzer-Brody S, Rubinow DR, Schmidt PJ. Reproductive aging, sex steroids, and mood disorders. Harv Rev Psychiatry 2009; 17(2): 87-102. 
3. Kapugama KG, Obadaarachchi LN, Ruwanpriya BV, Kuruppuarachchi KA. Depression presenting with genitalsymptoms - a series of unusual case histories. SLJ Psychiatry 2016; 7(2): 23-25.

4. Kuruppuarachchi KA, Wijeratne LT. The continuing story of dhat syndrome. Br J Psychiatry2004; 185(3): 260.

5. Jackson BT. A case of voyeurism treated by counter conditioning. Behav Res Ther 1969; 7(1): 133-4.

6. Mahadevan R, NikJaafar NR, Sidi H, Midin M, Das S. Is increased libido an atypical symptom of bipolar depression? An interesting case. J Sex Med 2013; 10(3): 883-6.

7. Anatale K, Kelly S. Factors influencing adolescent girls' sexual behavior: a secondary analysis of the 2011 youth risk behavior survey. Issues Ment Health Nurs 2015; 36(3): 217-21.

8. Fabre LF, Smith LC. The effect of major depression on sexual function in women. J Sex Med 2012; 9: 231-9.

9. Kalmbach DA, Kingsberg SA, Ciesla JA. How changes in depression and anxiety symptoms correspond to variations in female sexual response in a nonclinical sample of young women: A daily diary study. J Sex Med 2014; 11: 2915-27.

10. Hori H, Yoshimura R, Nakamura J. Increased libido in a woman treated with fluvoxamine: a case report. Acta Psychiatr Scand 2001; 103(4): 312-3. 